\section{Formation of a complex between HD-GYP, GGDEF and PilZ domain proteins regulates motility in Xanthomonas campestris}

\author{
Shi-qi An, ${ }^{1}$ Ji-liang Tang ${ }^{2}$ \\ ${ }^{1}$ Wellcome-Wolfson Institute for \\ Experimental Medicine, Queen's \\ University, Belfast, UK; ${ }^{2}$ State Key \\ Laboratory for Conservation and \\ Utilization of Subtropical Agro-biore- \\ sources, College of Life Science and \\ Technology, Guangxi University, \\ Guangxi, China
}

\begin{abstract}
RpfG is a member of a class of wide spread bacterial two-component regulators with an HD-GYP cyclic di-GMP phosphodiesterase domain. In the plant pathogen Xanthomonas campestris pv. campestris $(X c c)$, RpfG together with the sensor kinase $\mathrm{RpfC}$ regulates the synthesis of a range of virulence factors as a response to the cellcell Diffusible Signaling Factor (DSF). RpfG regulates many different virulence factors by divergent pathways. Physical interaction of RpfG with two diguanylate cyclase (GGDEF) domain proteins controls motility. This is a dynamic interaction that depends upon DSF signaling and involves the conserved GYP motif in the HD-GYP domain. Here we use synthetic peptide overlay technology and yeast two-hybrid analysis in conjunction with alanine substitution mutagenesis to define a motif within the GGDEF domain proteins required for interaction. We show that regulation of motility by the GGDEF domain proteins depends upon this motif. Furthermore, we show by $\mathrm{Y} 2 \mathrm{H}$ that both GGDEF domain proteins bind a specific PilZ domain adaptor protein, and this interacts with the pilus motor proteins PilU and PiIT. The results support a model in which DSF signaling influences motility through the interaction of proteins that affect pilus action. The motif required for HD-GYP domain interaction is conserved in a number of GGDEF domain proteins, suggesting that regulation via interdomain interactions may be of broad relevance.
\end{abstract}

\section{Introduction}

Cyclic di-GMP is now recognised as an almost universal second messenger in eubacteria that acts to regulate a wide range of functions including developmental transitions, adherence to surfaces, biofilm formation, motility and the synthesis of virulence factors. ${ }^{1-4}$ Cyclic di-GMP is synthesised from two GTP molecules by diguanylate cyclases (DGCs) that have a GGDEF domain and degraded by phosphodiesterases (PDEs) with either an EAL or HD-GYP domain. ${ }^{1}$ Cyclic di-GMP exerts a regulatory action through binding to diverse receptors that include a small adaptor protein domain called PilZ, transcription factors, riboswitches and enzymatically inactive variants of GGDEF, EAL or HD-GYP domain proteins. ${ }^{3,5,6}$ The multiplicity of GGDEF, EAL and HD-GYP proteins together with a range of receptors within the same bacterial cell indicate the considerable complexity in the organization of cyclic diGMP signaling which is relatively poorly understood. ${ }^{1}$

In the plant pathogen Xanthomonas campestris pv. campestris (Xcc), a twocomponent system comprising the sensor kinase $\mathrm{RpfC}$ and regulator $\mathrm{RpfG}$ is implicated in perception and signal transduction of the cell-to-cell signal Diffusible Signaling Factor (DSF). ${ }^{7-9}$ RpfC is a complex sensor kinase with a predicted membrane-associated sensory input domain. $\mathrm{RpfG}$ is a regulator with a CheY-like receiver (REC) domain attached to an HD-GYP domain. Mutation of $r p f G$ or $r p f C$ in $X c c$ leads to a coordinate reduction in the synthesis of virulence factors, including extracellular enzymes and the extracellular polysaccharide xanthan; alterations in biofilm formation; reduced pilus-dependent motility and a reduction in virulence. ${ }^{8} \mathrm{RpfG}$ regulates synthesis of these different factors by different mechanisms. Regulation of synthesis of extracellular enzymes, for example, involves the cyclic di-GMP responsive transcriptional regulator Clp, ${ }^{10,11}$ but not the physical interaction of RpfG with certain GGDEF domain-containing proteins needed to control pilus-dependent motility. These protein-protein interactions depend upon the GYP motif of the HD-GYP domain and are promoted by addition of the DSF signal. ${ }^{8}$

The residues of the GGDEF domain determining the specificity of interaction with the GYP motif of RpfG and the mechanisms by which inter-domain interactions regulate motility are unknown. In 2011, it was reported a specific motif was required for HD-GYP domain to interact with in a number of GGDEF domain proteins. ${ }^{12}$ However, this paper was recently retracted due to errors in data presentation. ${ }^{13}$ Here, we report on the outcomes of repeated key experiments from this study. We used syn-
Correspondence: Shi-qi An, WellcomeWolfson Institute for Experimental Medicine, Queen's University Belfast, Health Sciences Building, 97 Lisburn Road, Belfast, BT9 7BL, UK. E-mail: s.an@qub.co.uk

Ji-liang Tang, State Key Laboratory for Conservation and Utilization of Subtropica Agro-bioresources, College of Life Science and Technology, Guangxi University, 100 Daxue Road, Nanning, Guangxi 530004, China. E-mail: jltang@gxu.edu.cn

Key words: Cyclic di-GMP; Motility; Xanthomonas.

Contributions: the authors contributed equally.

Acknowledgements: we thank Robert Ryan, Max Dow and Delphine Caly for original data that promoted the study, helpful discussions and critical reading of the manuscript. We also must thank Rosemary O'Connor for initial data from the original study.

Conflict of interest: the authors declare no potential conflict of interest.

Funding: Ba Gui Scholar Program of Guangxi Zhuang Autonomous Region of China (2014A002).

Received for publication: 22 January 2018.

Revision received: 28 February 2018.

Accepted for publication: 13 March 2018.

This work is licensed under a Creative Commons Attribution NonCommercial 4.0 License (CC BY-NC 4.0)

(C) Copyright S. An, J. Tang, 2018

Licensee PAGEPress, Italy

Microbiology Research 2018; 9:7601

doi:10.4081/mr.2018.7601

thetic peptide overlay technology (SPOT) and yeast two-hybrid ( $\mathrm{Y} 2 \mathrm{H})$ analysis in conjunction with alanine substitution mutagenesis to define a motif within the GGDEF domain proteins required for interaction. We show that the action of the GGDEF domain proteins in regulation of motility depends on the motif required for RpfG interaction, but does not depend upon their enzymatic activity in cyclic di-GMP synthesis. Moreover, we show that both GGDEF domain proteins interact with a specific PilZ domain protein that also interacts with the pilus motor proteins PilU and PiIT. The results support a model in which DSF signaling influences motility at least in part through the interaction of proteins that affect pilus action.

Importantly, we found that this motif required for HD-GYP domain interaction is 
conserved in a select number of GGDEF domain proteins, suggesting that regulation via interdomain interactions may be important in a wide range of bacterial species and could allow parallel or co-ordinate regulation of processes within the cell that are independently controlled by different GGDEF domain proteins. Additionally, the non-enzymatic roles for enzymatically active GGDEF domain proteins highlight the potential for diverse regulatory actions of these proteins.

\section{Materials and Methods}

\section{Bacterial Strains, plasmids, and cul- ture conditions}

$X c c$ strains and culture conditions have been described previously. ${ }^{7,8}$ Most experiments were carried out in NYGB medium, which comprises $5 \mathrm{~g} \mathrm{liter}^{-1}$ bacteriological peptone (Oxoid, Basingstoke, U.K.), $3 \mathrm{~g}$ liter ${ }^{-1}$ yeast extract (Difco), and $20 \mathrm{~g} \mathrm{liter}^{-1}$ glycerol. For biofilm formation, Xcc was grown in L medium, which comprises $10 \mathrm{~g}$ liter-1 bactotryptone (Difco), $5 \mathrm{~g} \mathrm{liter}^{-1}$ yeast extract, $5 \mathrm{~g} \mathrm{liter}^{-1}$ sodium chloride, and $1 \mathrm{~g}$ liter $^{-1} \mathrm{D}$-glucose. $E$. coli strains were grown in $\mathrm{LB}$ medium at $37^{\circ} \mathrm{C}$. Other plasmids and strains used are shown in Table S1. Where required antibiotics were used at concentrations of $100 \mathrm{mg} \mathrm{ml}^{-1}$ for ampicillin, $50 \mathrm{mg}$ $\mathrm{ml}^{-1}$ for rifampicin and $20 \mathrm{mg} \mathrm{ml}^{-1}$ kanamycin and $15 \mathrm{mg} \mathrm{ml}^{-1}$ tetracycline.

\section{Molecular genetic techniques}

All standard genetic techniques were performed as described by Sambrook et al. ${ }^{14} \mathrm{Pfu}$ polymerase (Promega ${ }^{\mathrm{TM}}$ ) was used for all PCR reactions. Primers were synthesized by Sigma-Genosys. Amplified fragments were cloned using the TOPO TA cloning kit (Invitrogen ${ }^{\mathrm{TM}}$ ). The identity of each cloned fragment was confirmed by sequencing by AGOWA genomics (Berlin, Germany).

\section{Yeast two-hybrid (Y2H) screen}

The methods for construction and analysis of the $X c c$ genomic DNA prey library in the plasmid vector pOAD were as described previously. 8,15 Xcc DNA sequence coding for the GGDEF domain proteins (XC_0249 or XC_0420) or PilZ protein (XC 2249 ) used as bait were amplified by polymerase chain reaction (PCR) using $X c c$ genomic DNA as template and primers designed based on the $X c c$ genome sequence. Primer sequence information is available upon request. These primers were designed to contain unique restriction sites to facilitate cloning into the pOBD vector downstream of the Gal4 DNA binding domain. After transformation into $E$. coli
DH5a cells, individual colonies were picked for plasmid isolation and confirmation by DNA sequencing. The bait construct was transformed into S. cerevisiae AH109 and this strain was used to screen the Xcc genomic DNA prey library. Approximately 2x108 yeast transformants (carrying both and prey vectors) were plated on selective media that was lacking adenine, histidine, tryptophan and leucine. 1,500 transformants displaying prototrophy were transferred plates with adenine and histidine but lacking tryptophan and leucine. Preys were identified by isolation of plasmids and sequencing. For measurement of interactions of the HD-GYP domain and its variants with GGDEF domains from specific $X c c$ proteins, doubly transformed yeast strains were grown in YPD medium and beta-galactosidase assays (reflecting GAL4 activation) were performed as described by. In addition, transformants grown in nonselective media to an OD of 0.3 were plated on selective media and the number of colonies that appeared was counted. This gives a second correlative assessment of the strength of interaction reflecting the degree of activation of $L E U, H I S, T R P$ and $A D E$.

\section{Site directed mutagenesis of GGDEF or PilZ domain containing proteins}

Site-directed mutagenesis to introduce the alanine alterations by using mutagenic PCR in a two-step protocol as previously reported. ${ }^{8,15}$ In the first round of PCR, two separate reactions were carried out with one of a pair of primers of complementary sequence carrying the desired alteration and the HDGYPHis 6 construct in pLAFR3 as template. The products of the first round of PCR were used as templates for a second round of PCR (Mutagenic primer sequences will be supplied upon request).

\section{Construction of protein expression vectors}

PCR fragments coding for the GGDEF domains from the Xcc proteins [XC_0249 or XC_0420] or PilZ domain protein [XC_2249] were cloned into pET-28a for expression as His-tagged fusions or pGEX4T-1 when a glutathione $S$-transferase (GST) tag is required. All the plasmid constructs were confirmed by DNA sequencing. DNA fragments coding $\mathrm{XC}_{-} 1359$ (PilU), XC 1060 (PilB) and $\mathrm{XC}^{-} 1358$ (PilT) were amplified by PCR using Xcc genomic DNA as template. After digestion with specific restriction enzymes, the PCRamplified fragments were inserted into $\mathrm{pET}$ $28 \mathrm{a}$ (Novagen $^{\mathrm{TM}}$ ) as described previously. 8,15

\section{Protein purification}

His-tagged proteins were purified by nickel affinity chromatography as described previously. ${ }^{16}$ For purification of the genes that were fused to the GST gene gel filtration was carried using a Sephadex G-75 (Sigma) as previously described. ${ }^{16}$ In all cases protein concentration was assayed using NanoDrop ${ }^{\circledR}$. Purified proteins were stored at $-20^{\circ} \mathrm{C}$.

\section{Far Western in vitro protein-protein interaction assays}

In vitro binding assays were performed as described previously. ${ }^{15}$ Briefly, approximately $20 \mathrm{mg}$ of purified recombinant proteins or total lysates of $E$. coli cultures expressing recombinant proteins were resolved by SDS-PAGE (12\% acrylamide) and transferred to nitrocellulose membranes. The membranes were blocked with TBS-TTmilk [140 mM NaCl, $20 \mathrm{mM}$ TrisHCl (pH 7.4), 0.1\% Triton X-100, 0.1\% Tween-20, 5\% powdered milk] for $1 \mathrm{~h}$ and then probed with anti-His6 antiserum as described previously. ${ }^{15}$

\section{Cyclic di-GMP binding assay}

Assessment of cyclic di-GMP binding was carried out using a variation of the method used previously. ${ }^{16}$

\section{Phenotypic analysis}

All phenotypic assays of Xcc strains used in this study have been described previously. Activities of the extracellular enzymes b-1,4-endoglucanase and b-1,4mannanase in culture supernatants were estimated by radial diffusion assays into substrate-containing plates by using locust bean gum (Sigma) and carboxymethylcellulose. Motility was assayed as described previously on NYGB solidified with $0.5 \%$ Eiken agar (Eiken Chemical, Tokyo)

\section{Results}

\section{Identifying residues required for HD-GYP::GGDEF domain interac-} tions by peptide array screening

In previous work it was shown that the HD-GYP domain of RpfG interacted with eight GGDEF domain proteins (XC_0249, XC_0420, XC 0613, XC 0675, XC_1803, XC_2274, XC__2795 and XC_2866) as assessed by yeast two hybrid analysis, whereas full length RpfG only interacts with two of these proteins, XC_0249 and XC_ 0420, when analysed by FRET in Xcc. ${ }^{8}$ To examine the potential residues in the GGDEF domain that may be important for this interaction, we screened a peptide array with purified proteins XC_0249 and 
XC_0420 with HD-GYPHis6, ADGYPHis6, HD-AAAHis6 and RpfGHis6. The peptide arrays comprised of immobilised overlapping peptides of 25 amino acids in length, which are offset by 5 amino acids (Figure 1A) and interactions were monitored with a specific antibody to the His6 tag. The screening of the XC_0420 peptide array identified the sequence MLDLDD as a possible site of interaction (Figure 1A).

To provide further evidence of a role for these residues, alanine substitutions were introduced within the peptide sequence TRTQRSGQPLCVAMLDLDDFRRLNET. These alanine substitutions alter the binding of the HD-GYP domain, consistent with the role of these residues in protein-protein interaction (Figure 1B). Alignment of the GGDEF domains of the eight interacting proteins identified by $\mathrm{Y} 2 \mathrm{H}$ suggested the presence in each amino acid sequence of a conserved DxD motif that was in an equivalent position relative to the active site (Asite) motif (Figure 1C).

\section{The DxD motifs role in protein-pro- tein interactions}

The role of the DxD motif in in vivo interaction between RpfG and different GGDEF domain proteins was assessed using $\mathrm{Y} 2 \mathrm{H}$ analysis. Here the HD-GYP domain of RpfG was used as bait and the GGDEF domains of XC_0420 and XC_0249 as prey (Figure 2). Interaction of the HD-GYP domain with the GGDEF-domains from XC_0420 (A) and XC_0249 (B) was assessed. Interestingly, the interactions were significantly reduced by alanine substitution in the $x x D x D x$ site. However, no effects were seen when alanine substitution was carried out in the I-site (RxxD) or A-site (GGDEF) of either XC_0249 (Figure 2C, D) or XC_0420 (not shown). As expected, alanine substitutions in the GGDEF motif abrogated the activity of the XC_0249 and XC_0420 as diguanylate cyclases (Figure S1). However, alanine substitution in the $x x D x D x$ motif had no influence on diguanylate cyclase activity (Figure S1). Overall these observations imply a role for the DxD motif in protein interaction in vivo.

The interaction motif is required for the regulatory action of the GGDEF domain targets on motility

Although mutation of the genes encoding either XC_0249 or XC_0420 has unaltered motility, the XC_0249XC_0420 double mutant has severely reduced motility, a phenotype that is also seen for the $\operatorname{rpf} G$ mutant $^{8}$ (Figure 3A). Motility can be restored to this double mutant by expression
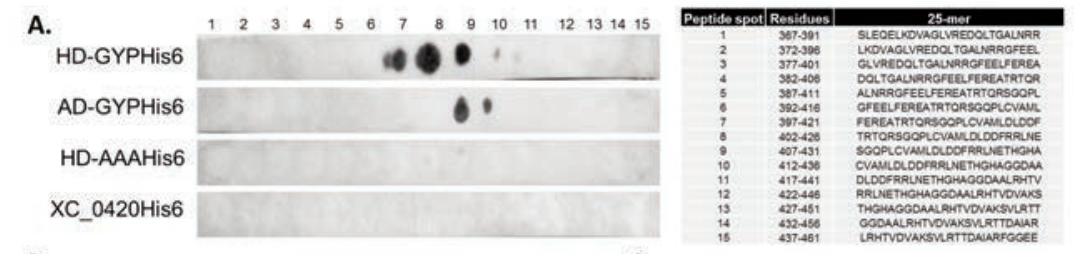

B. $\quad \begin{array}{llllllllllllll}1 & 2 & 3 & 4 & 5 & 6 & 7 & 8 & 9 & 10 & 11 & 12 & \text { C. }\end{array}$

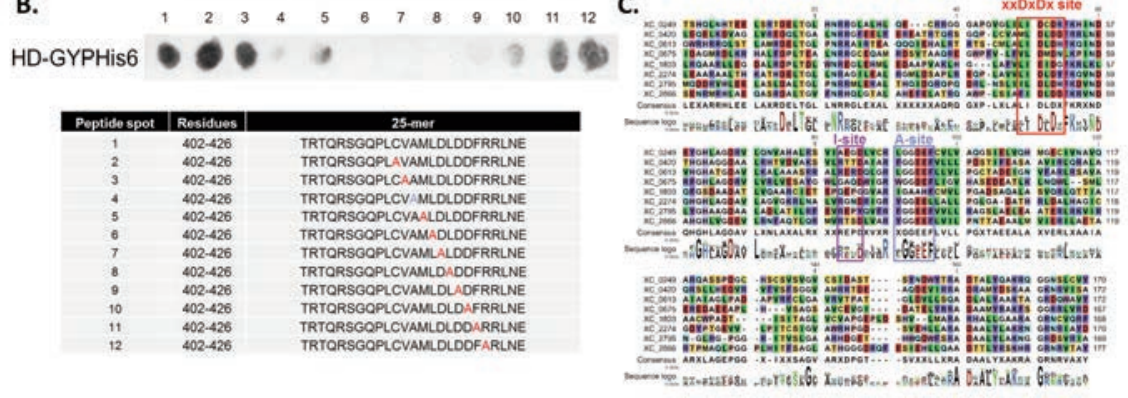

Figure 1. Peptide array and alanine scanning used to identify residues required for HDGYP::GGDEF-domain interaction. (A) Purified HD-GYPHis6, AA-GYPHis6, HDAAAHis6 and XC_0420His6 was used to probe an array of immobilised peptide spots of overlapping 25-mer peptides each offset by five amino acids of the entire XC_0420 sequence. Table shows the spot numbers and relate to sequence of each peptide. Immunoblotting was used to detect interactions. (B) Arrays in which the 25 amino acids in peptide 8 of XC_0420 were sequentially substituted with alanine and probed with HDGYPHis6. In the case where the parent amino acid was alanine, it was substituted by aspartate. The decrease in intensity in binding is indicative of the role of the sequence LCVAMLDLDD of the GGDEF domain of XC_0420 in interaction. (C) Amino acid sequence comparisons between the GGDEF domains interacting RpfG as identified by $\mathrm{Y} 2 \mathrm{H}$. The sequences were aligned using ClustalW. The positions of the conserved xxDxDx site and A-site (GGDEF or GGEEF motif) in each amino acid sequence are indicated. Also indicated is the position of the I-site ( $\mathrm{xxD}$ motif); this motif is not present in all of the sequences.
A.

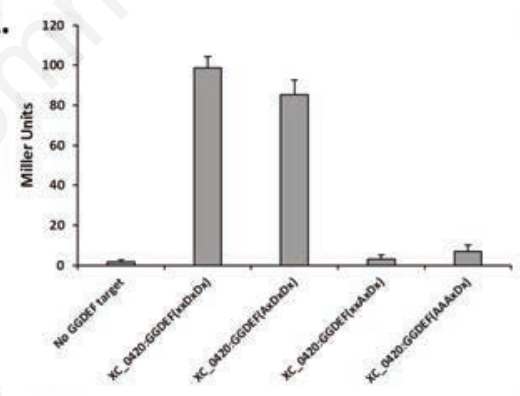

C.

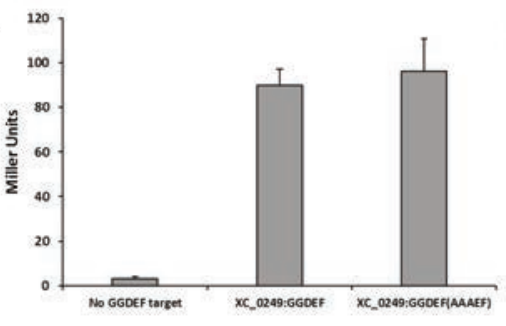

B.

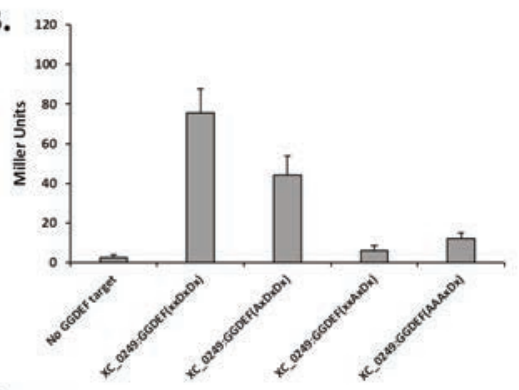

D. ${ }^{120}$

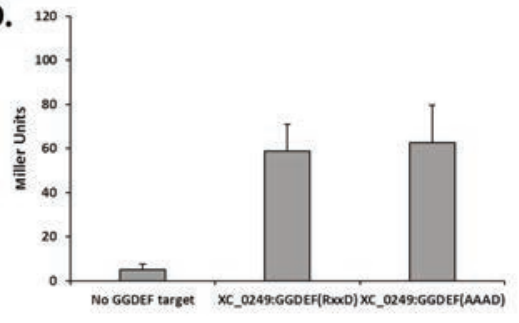

Figure 2. HD-GYP-GGDEF interactions as measured by $\mathrm{Y} 2 \mathrm{H}$ depend upon the $\mathrm{xxDxDx}$ site. The full-length XC_0420 and XC_0249 proteins, their GGDEF domains and different variants were assessed. The GGDEF domain of XC_0249 and XC_0420 protein or the HD-GYP domain of RpfG was generated. These constructs were used to measure interactions in vivo by $\mathrm{Y} 2 \mathrm{H}$. Interaction of the HD-GYP domain with the GGDEF-domains from XC_0249 (A) or XC_0420 (B) is affected by alanine substitution in the $\mathrm{xxDxDx}$ site. However, no affects were seen when alanine substitution was carried out in the I-site (RxxD) or A-site (GGDEF) of the XC_0249 or XC_0420 (C, D). Values given are means \pm SD of triplicate measurements. 
of a single or tandem GGDEF domain from XC_0249 and/or XC_0420, each carrying His $\overline{6}$ tags (Figure 3B). Expression of variants with alanine substitution in the $\mathrm{xxDxDx}$ site was not, however, able to restore motility to the $X C \_0249 X C \_0420$ double mutant (Figure 3B). Importantly, Western analysis with an anti-His6 antiserum demonstrated that all variant proteins were expressed in Xcc (Figure 3C). Together, the findings revealed that the regulatory activity of these proteins on motility does require protein-protein interaction with RpfG.

Further interaction partners for the GGDEF domain proteins XC_0249 and XC_0420

To identify proteins that could connect the formation of the HD-GYP::GGDEF complex to modulation of motility, $\mathrm{Y} 2 \mathrm{H}$ analysis was employed with the GGDEF domains from XC_0249 and XC_0420 as baits. Among the interacting proteins identified in these screens was a PilZ domaincontaining protein XC 2249, which interacted with both $\mathrm{XC} 0249$ and XC 0420 baits (Table S2). The $\overline{\mathrm{Y}} 2 \mathrm{H}$ analysis observations were supported by Far-western blotting experiments in which lysates of bacteria expressing the PilZ domain protein were separated by SDS polyacrylamide gel electrophoresis, transferred to nitrocellulose membranes and then surveyed with the His6 tagged GGDEF domain of XC_0249 or XC_0420. These domains were subsequently detected with an anti-His6 antiserum. Three other PilZ domain proteins of Xcc (XC_0965, XC_3221 and XC_2317) were also included to assess if this interaction was general or specific (Figure 4A, B). Only XC_2249 appeared to give any signals, suggesting that the GGDEF domains of XC_0249 and XC_0420 interact only with this specific $\mathrm{PilZ}$ domain protein. Interestingly, XC_2249 is involved in regulation of motility and plant virulence in Xcc. ${ }^{17}$ HD-GYP, GGDEF and PilZ domain protein interplay was further examined by $\mathrm{Y} 2 \mathrm{H}$ experiments where the intensity the interactions between proteins was quantified. A strong interaction was seen between the HD-GYP and the GGDEF domains (Figure 4C); this interaction did not require the GGDEF motif. Other interactions were also seen between the GGDEF domains and the PilZ protein XC_2249 (Figure 4D), as expected from the screen reported above. However, simultaneous expression of the HD-GYP domain enhanced this interaction. By contrast, there was only a weak interaction between the HD-GYP domain and the PilZ domain protein in the absence of the GGDEF domain (Figure 4D).
A.

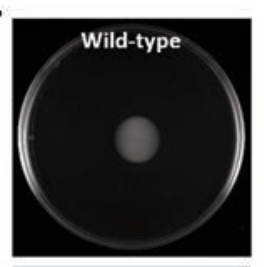

B.
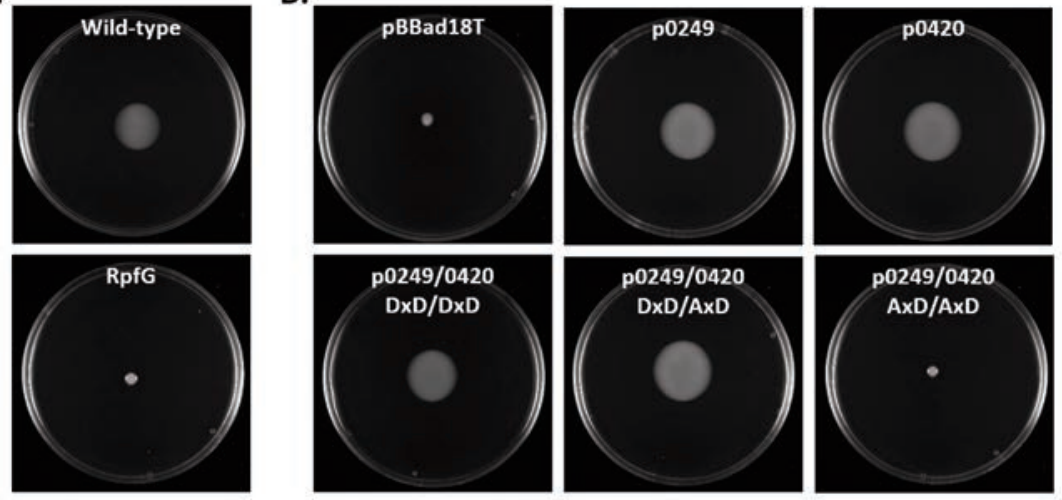

C.

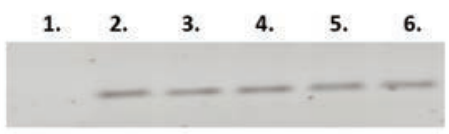

Figure 3. The regulatory activity of the GGDEF-domain proteins XC_0249 and XC_0420 on motility is dependent on the $\mathrm{xxDxDx}$ site. Although mutation of the genes encoding $X C \_0249$ and $X C \_0420$ alone has no effect on motility, the XC_0249/XC_0420 double mutant has the highly reduced motility seen with the $r p f G$ mutant (compare panels $A$ and B). (B) Motility is restored to the $X C_{-} 0249 / X C_{-} 0420$ double mutant by single (p0249) or tandem (p0249/0420) expression of their GGDEF domains carrying His6 tags. However, expression of the variant GGDEF domains with alanine substitutions in the $\mathrm{xxDxDx}$ site did not restore motility. pBBad18T represents the empty vector control (C). Western analysis demonstrated that the variant proteins were expressed in Xcc.

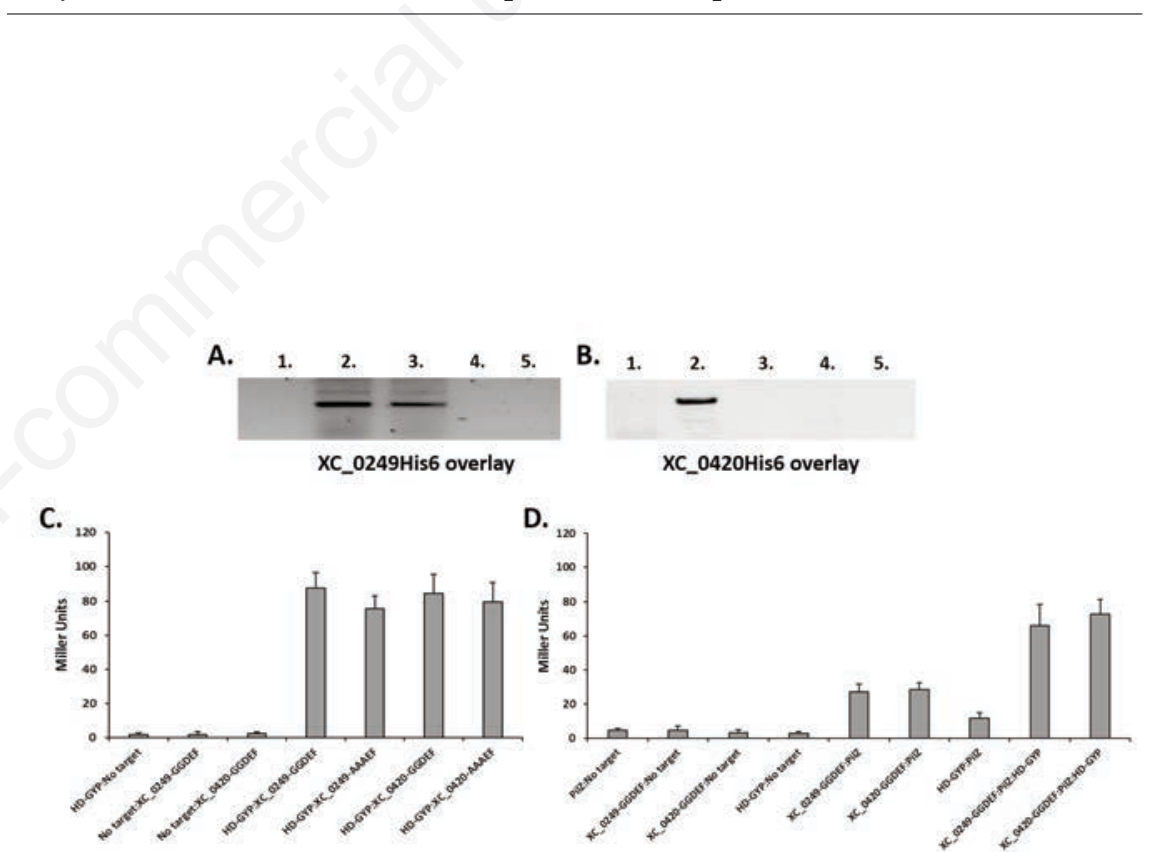

Figure 4. Far-Western analysis showing interactions of specific PilZ domain proteins with GGDEF domains from XC_0249 and XC_0420 proteins. Lysates of Xcc expressing the different PilZ domains (XC__0965, XC_0249, XC_2317, XC_3221) were separated by SDS/PAGE, transferred to nitrocellulose membranes, and overlaid with $50 \mu \mathrm{g}$ of XC_0249His6 (A) or XC_0420His6 (B). Binding was detected with an anti-His6 antiserum. Lanes on left hand side image: (1) H2O control; (2) XC_2249; (3) XC_2249; (4) XC_0965; (5) XC_3221. Lanes on right hand side image: (1) H2O control; (2) XC_2249; (3) XC_2317; (4) XC_0965; (5) XC_3221. HD-GYP-GGDEF-PilZ protein interactions as revealed by $\mathrm{Y} 2 \mathrm{H}(\mathrm{C})$. For these experiments, the HD-GYP domain of RpfG, the GGDEF domain of XC_0420, and the full-length PilZ protein XC_2249 were expressed. No signal of interact was seen unless all three proteins were expressed. No signal was seen when HD-GYP domain and PilZ were expressed alone, suggesting no direct interaction between them. Values given are means \pm SD of triplicate measurements. 


\section{XC_2249 binds cyclic di-GMP but} this action is not required for motility

PilZ domain proteins are known to bind cyclic di-GMP and can be responsible for their ability to exert a regulatory action., 3,5,6 Purified XC_2249 showed that this protein had the ability to bind cyclic di-GMP (Figure 5A). The binding of nucleotide was prevented by alanine replacement of R21 and Q24, the conserved residues within PilZ domain proteins implicated in binding (Figure 5A). To determine if regulation of motility was dependent on cyclic di-GMP binding, the effects of expression of an R21AQ24A variant of XC_2249 in the $X C \_2249$ mutant background was examined. This XC 2249 variant protein reestablished motility in the XC_2249 mutant (Figure 5B), indicating that nucleotide binding by XC_2249 is not needed for motility under these conditions. Importantly, Western analysis showed that the both wild type and variant proteins of XC_2249 were expressed similarly in Xcc (Figure 5C).

The action of XC_0249 and XC_0420 in regulation of motility does not depend upon the enzymatic activity

The proteins XC_0249 and XC_0420 are active as $\mathrm{DGCs}^{8}$ and alterations within the GGDEF motif inactivate these proteins as enzymes (Figure S1). The observation that XC_2249 does not apparently require cyclic di-GMP binding to regulate motility prompted us to examine if DGC activity of XC_0249 and XC_0420 was needed for control of motility. Intriguingly, the expression of enzymatically inactive AAAEF variants of the XC_0249 and XC_0420 in the XC_0249/XC_0420 double mutant still allowed restoration of motility (Figure S2 and data not shown). These findings indicated that the control of motility by XC_0249 and XC_0420 requires protein contact with RpfG but not their activity as diguanylate cyclase enzymes.

The PilZ domain protein XC_2249 interacts with the pilus motor proteins PilU and PilT

To identify possible targets for XC_2249 action a further Y2H screen with XC_2249 as bait was carried out. This screen recognized the pilus motor proteins PilT and PilU as potential interacting proteins (Table S3). These proteins have been previously implicated in pilus retraction. ${ }^{18-}$ ${ }^{20}$ The Y2H findings were extended by Farwestern blotting experiments. For these experiments, lysates of bacteria that were over-expressing the selected proteins were separated by SDS PAGE, transferred to
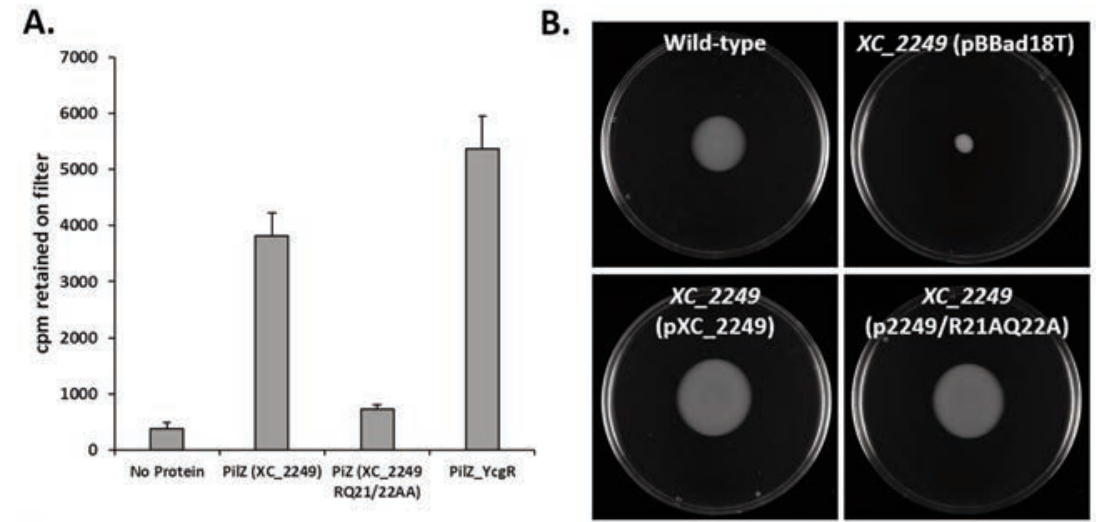

c.

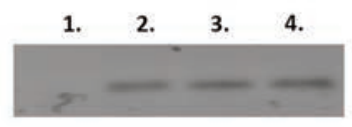

Figure 5. The binding of cyclic di-GMP by the PilZ-domain protein XC_2249 is not required to regulate motility in Xcc. (A) Cyclic di-GMP binds to XC_2249His6 and PilZ_YcgR (positive control) but not to the R21AQ22A variant of XC_2249His6. All reactions contained $10 \mu \mathrm{M}\left[{ }^{32} \mathrm{P}\right]$-cyclic-di-GMP. (B). Alanine substitution at two positions $(\mathrm{R} 21 \mathrm{Q} 22)$ in the PilZ domain protein XC_2249 that are implicated in cyclic diGMP binding has no effect on the ability to restore motility to a pilZ (XC_2249) mutant. Top left: wild type; top right XC2249/pBBad18T vector; bottom left: $X C_{\_} 2249 / \mathrm{pXC} \_2249$; bottom right: $X C \_2249 / \mathrm{pXC} \_2249$ encoding the R21AQ22A variant $(C)$. Western analysis demonstrated that these variant proteins are expressed in Xcc. Lanes (1) H2O control; (2) WT (pXC_2249His6); (3) XC_2249 (pXC_2249His6) and (4) XC_2249 (pXC_2249His6-R21AQ22A).

A.
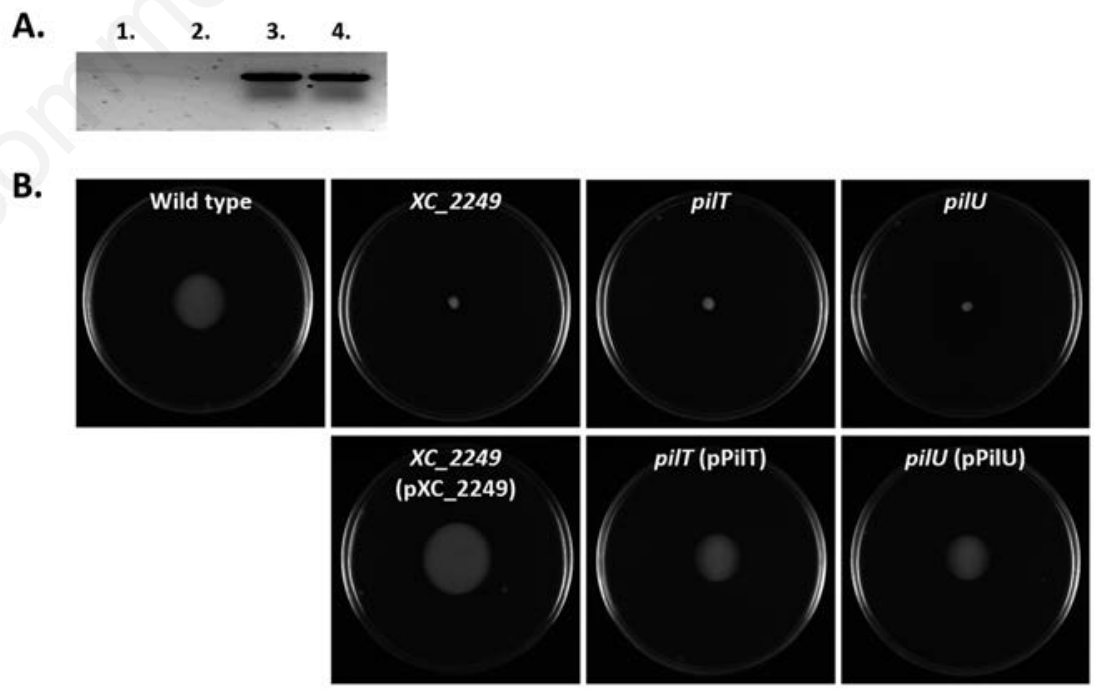

Figure 6. The PilZ-domain protein XC_2249 interacts with the pilus motor proteins PilT and PilU. (A) Far-Western analysis showing interactions of the PilZ-domain protein XC_2249 with pilus motor proteins PilT and PilU. Lysates of Xcc expressing the PilZdomain protein XC_2249 were separated by SDS-PAGE, transferred to nitrocellulose membranes and overlaid with $50 \mathrm{Jg}$ of PilTHis 6 or PilUHis6. Binding was detected with an anti-His6 antiserum. Lanes: (1) $\mathrm{H} 2 \mathrm{O}$ control (2) XC_2249, (3) XC_1358, (4) XC_1359. Lanes: (1) H2O control (2) XC_2249, (3) XC_2317, (4) XC_3221. (B) Mutation of genes encoding encoding PilZ, $\overline{\mathrm{P} i l T}$ and PilU gives rise to similar altered motility phenotypes on $\mathbf{0 . 5 \%}$ Eiken agar-NYG plates. Expression of the wildtype copy of the gene with a His 6 tag in the corresponding mutant restores motility to that seen in the wild type 8004 with the empty pLAFR3 vector. 
nitrocellulose membranes and probed with a His6 tagged XC_2249. Signals for both target proteins were detected using an antiHis6 antiserum (Figure 6A). The $\mathrm{Y} 2 \mathrm{H}$ and Far western analyses were repeated using PilB, which is associated with pilus polymerization; there was however no evidence for interaction with XC 2249. Mutational analysis revealed that pilT and pilU strains have reduced motility, to a level comparable with the $r p f G$ mutant and the $X C \_0249 X C \_0420$ double mutant (Figure $6 \mathrm{~B})$. These observations are consistent with a model by which the PilZ domain protein interaction with PilU and PilT is dependent on the interaction between the GGDEF domain proteins and the specific PilZ domain protein.

\section{Discussion and Conclusions}

In the current study we examined the mechanisms underlying signal transduction in the DSF/rpf cell-cell signaling system. As was previously showed, DSF signaling promotes the formation of a complex between the HD-GYP domain response regulator RpfG and two GGDEF domain proteins to regulate pilus-dependent motility. ${ }^{8,12}$ It is thought that this interaction, which requires the GYP motif in the HD-GYP domain protein, is promoted when the REC domain of RpfG is phosphorylated in response to DSF recognition. ${ }^{8,12}$

Here we show that the interaction requires the site $\mathrm{xxDxDx}$ containing the motif DxD that occurs in a number of GGDEF domain containing proteins. Two of these GGDEF domain proteins (XC_0249 and XC_0420) interact both with $\mathrm{RpfG}$ and with a PilZ domain protein (XC 2249), previously implicated in the control of motility in Xcc. ${ }^{17}$ We further show that XC_2249 interacts with the pilus retraction proteins PilU and PilT. Together the findings are consistent with a model whereby a tri-protein complex of HD-GYP, GGDEF and PilZ domain proteins interacts with PilU and PilT to manipulate pilus function with a consequent impact on bacterial motility.

We also show that although the PilZ domain protein XC_2249 binds the nucleotide cyclic di-GMP, this binding is not needed for the control of motility. Work in an associated bacterium Xanthomonas axonopodis pv. citri has shown that a different PilZ domain protein (XAC1133) is also unable to bind cyclic di-GMP but interacts with both PilB (an ATPase required for pilus polymerization) and an the EAL domain of FimX (an GGDEF-EAL domain protein) which regulates type IV pilus local- ization and biogenesis in other bacterial species including X.citri. ${ }^{20}$ FimX has a degenerate GGDEF domain that appears to have no PDE activity but retains the ability to bind cyclic di-GMP both in the presence and absence of PilZ (XAC1133). Homologues of FimX, PilZ (XAC1133) and PilB occur in Xcc. Our current work, taken together with these previously published observations, suggest the occurrence of multiple mechanisms of regulation of pilus action that may be responding to very different stimuli (Figure S3).

Cyclic di-GMP binding is not needed for XC_2249 action in pilus-dependent motility in $X c c$, suggesting that the mechanism of action is different from that of the highly studied PilZ domain protein YcgR that regulates swimming motility in Escherichia coli. ${ }^{21,22}$ Furthermore, although the GGDEF domain proteins XC_0249 and XC_0420 exhibit DGC activity, this is not needed for their regulation of motility in Xanthomonas. This does not rule out the likelihood that these proteins have other regulatory actions that depend upon modulation of cyclic di-GMP levels.

The DxD motif, which is shown to be important for RpfG-GGDEF interaction by SPOT array, occurs in an equivalent location in XC_0249 and XC_0420. This motif appears to be distinct from the catalytic A site and I site $(\mathrm{RxxD})$ in the linear sequence alignment, although only resolution of the three-dimensional structure of these proteins will reveal the exact spatial relationship between these different sites. However, for now it appears that the catalytic A site and I site $(\mathrm{RxxD})$ are not involved in interaction with the HD-GYP domain of RpfG. Inspection of the sequences of GGDEF domain proteins from a collection of wide ranging bacteria reveals conservation of the DxD motif. Furthermore, it appears that the presence of this DxD motif in GGDEF domain proteins is correlated with the incidence of HD-GYP domain proteins in bacteria. This conservation of the DxD motif and HD-GYP domain proteins suggests that interaction of GGDEF domain proteins with HD-GYP domain proteins might of broad relevance.

\section{References}

1. Hengge R. Principles of c-di-GMP signalling in bacteria. Nat Rev Microbiol 2009; 7:263-73

2. Schirmer T, Jenal U. Structural and mechanistic determinants of c-di-GMP signalling. Nat Rev Microbiol 2009; 7:724-35

3. Sondermann H, Shikuma NJ, Yildiz FH.
You've come a long way: c-di-GMP signaling. Curr Opin Microbiol 2012;15:140-6.

4. Römling U, Galperin MY, Gomelsky M. Cyclic di-GMP: the first 25 years of a universal bacterial second messenger. Microbiol Mol Biol Rev 2013;77:1-52.

5. Ryan RP. Dynamic complex formation between HD-GYP, GGDEF and PilZ domain proteins regulates motility in Xanthomonas campestris. Mol Microbiol 2012;86:557-67.

6. Ryan RP, Tolker-Nielsen T, Dow JM. When the PilZ don't work: effectors for cyclic di-GMP action in bacteria. Trends Microbiol 2012;20:235-42.

7. Slater H, Alvarez-Morales A, Barber $\mathrm{CE}$, et al. A two-component system involving an HD-GYP domain protein links cell-cell signalling to pathogenicity gene expression in Xanthomonas campestris. Mol Microbiol 2000;38:986-1003.

8. Ryan RP. Cell-cell signal-dependent dynamic interactions between HD-GYP and GGDEF domain proteins mediate virulence in Xanthomonas campestris. Proc Natl Acad Sci USA 2010;107:5989-94.

9. Cai Z, Yuan ZH, Zhang H, et al. Fatty acid DSF binds and allosterically activates histidine kinase RpfC of phytopathogenic bacterium Xanthomonas campestris pv. campestris to regulate quorum-sensing and virulence. PLoS Pathog 2017;13:e1006304.

10. Chin KH. The cAMP receptor-like protein CLP is a novel c-di-GMP receptor linking cell-cell signaling to virulence gene expression in Xanthomonas campestris. J Mol Biol 2010;396:64662.

11. Tao F, He YW, Wu DH, et al. The cyclic nucleotide monophosphate domain of Xanthomonas campestris global regulator Clp defines a new class of cyclic diGMP effectors. J Bacteriol 2010;192: 1020-9.

12. Ryan RP, Dow JM. Communication with a growing family: diffusible signal factor (DSF) signaling in bacteria. Trends Microbiol 2011;19:145-52.

13. Ryan RP. Dynamic complex formation between HD-GYP, GGDEF and PilZ domain proteins regulates motility in Xanthomonas campestris. Mol Microbiol 2017;104:533.

14. Sambrook J, Fritsch EF, Maniatis T. Molecular cloning: a laboratory manual, 2nd ed. Cold Spring Harbor, NY: Cold Spring Harbor Laboratory Press; 1989. pp 10.51-67.

15. Andrade MO. The HD-GYP domain of RpfG mediates a direct linkage between 
the Rpf quorum-sensing pathway and a subset of diguanylate cyclase proteins in the phytopathogen Xanthomonas axonopodis pv citri. Mol Microbiol 2006;62:537-51.

16. Fazli M. The CRP/FNR family protein Bcam1349 is a c-di-GMP effector that regulates biofilm formation in the respiratory pathogen Burkholderia cenocepacia. Mol Microbiol 2011;82:32741.

17. McCarthy Y. The role of PilZ domain proteins in the virulence of
Xanthomonas campestris pv. campestris. Mol Plant Pathol 2008;9:819-24.

18. Mattick JS. Type IV pili and twitching motility. Annu Rev Microbiol 2002;56:289-314.

19. Craig L, Li J. Type IV pili: paradoxes in form and function. Curr Opin Struct Biol 2008;18:267-77.

20. Guzzo CR, Salinas RK, Andrade MO, Farah CS. PilZ protein structure and interactions with PilB and the FimX EAL domain: implications for control of type IV pilus biogenesis. J Mol Biol 2009;393:848-66.

21. Paul R, Weiser S, Amiot NC, et al. Cell cycle-dependent dynamic localization of a bacterial response regulator with a novel di-guanylate cyclase output domain. Genes Dev 2004;18:715-27.

22. Boehm A, Kaiser M, Li H, etal. Second messenger-mediated adjustment of bacterial swimming velocity. Cell 2010;141:107-16. 